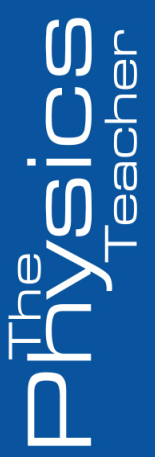

\title{
Improved demo of granular flow
}

Cite as: Phys. Teach. 58, 147 (2020); https://doi.org/10.1119/1.5145398

Published Online: 19 February 2020

Said Shakerin

ARTICLES YOU MAY BE INTERESTED IN

Breathtaking Physics: Human Respiration as a Heat Engine

The Physics Teacher 58, 150 (2020); https://doi.org/10.1119/1.5145400

Photoelectric effects

The Physics Teacher 58, 147 (2020); https://doi.org/10.1119/1.5145396

Rainbows: A Graphical Approach

The Physics Teacher 58, 152 (2020); https://doi.org/10.1119/1.5145401

\section{AAPT}

Advance your teaching and career as a member of AAPT 


\section{Photoelectric effects}

I was very pleased to see Josu Martinez-Perdiguero's article in the November 2019 issue. ${ }^{1}$ Its arrival was well timed as I had literally just finished my lecture on the photoelectric effect. The analysis was spot-on in stressing the difference between the "bang-for-the-buck" that each photon packs (as measured by its frequency) vs. the number of photons in a beam (as measured by the intensity). However, I prefer a different approach compared to the I-V curve (Figs. 2 and 3). I posed the following question to my students on a quiz supporting the photoelectric effect lecture.

Now let's talk photons. We know that the energy of a given photon is $E=h f$. Suppose that we have two laser beams of light-the first one is red $(600 \mathrm{~nm})$ and the second blue $(400 \mathrm{~nm})$. Further suppose that the energy output of each laser is identical at $1 \mathrm{~W}$ (i.e., $1 \mathrm{~J} / \mathrm{s}$ ). Answering only with red or blue,

1. Which beam has the most photons?

2. Which beam has the most energetic photons?

The guided conversation (usually with a dollar's worth of quarters and dimes to represent equal intensities of blue and red photons) that follows goes a long way to drive home the point Martinez-Perdiguero was at pains to make.

1. Josu Martinez-Perdiguero, "On a common mistake in the description of the photoelectric effect," Phys. Teach. 57, 536 (Nov. 2019).

Conrad Schiff

Capitol Technology University

\section{Starfish in the sky}

In 1972-73 I was on sabbatical leave at the Kingston, Jamaica, campus of the University of the West Indies. I taught a few classes and developed a series of demonstration lectures that were given on Saturday mornings for enrichment purposes. The two most popular were "Physics Without Friction" and "Suspension Bridges," and these were taken on tour throughout the island for school and teacher audiences. In the spring of 1973 I was sent to Georgetown in the Cayman Islands to give these two talks to general audiences. To cool down, I donned mask and fins and swam down about 10 feet to the sandy bottom of Seven Mile Beach. There was no wind and the surface of the sea was like glass. When I looked up, I could see starfish in the sky!

The figure shows what was happening. The starfish were actually on the bottom, and I was viewing them by reflection from the water surface. At an angle of about $30^{\circ}$ an appreciable amount of the light was reflected downward from the surface of the water. My mind could not fathom the idea that I was seeing a reflection. Instead, I assumed that I was seeing an object in front of me. This is the same idea behind the Pepper's Ghost Illusion ${ }^{1}$ and behind Lincoln's Visual Physics contribution. ${ }^{2}$

The Sun was nearly overhead, and the clearness of the water allowed objects on the bottom to be brightly illuminated. I suppose that the same effect can be seen in a swimming pool if the water surface is allowed to come to rest.

1. Thomas B. Greenslade Jr., "Pepper's Ghost," Phys. Teach. 49,

338-339 (Sept. 2011).

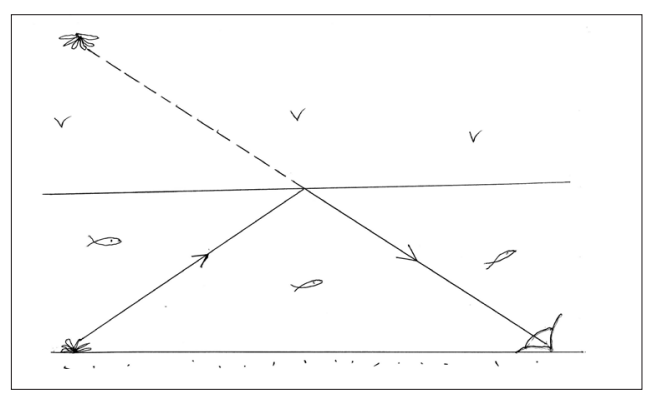

Fig. 1. How I saw the Starfish in the Sky. At the bottom is sand, in the middle is water, and at the top is sky.

2. James Lincoln, "Visual Physics: Total internal reflection," Phys. Teach. 52, 227 (April 2014).

Tom Greenslade Jr. Kenyon College, Gambier, $\mathrm{OH}$

\section{Improved demo of granular flow}

The purpose of this letter is to present an improved version of the eruption-like flow demonstration device that I had reported on in this publication. ${ }^{1}$ The earlier version dealt with only one granular material, ceramic microsphere (CMS). The improved version contains CMS and sand side by side for comparison as shown below. Their average grain sizes differ by approximately an order of magnitude.

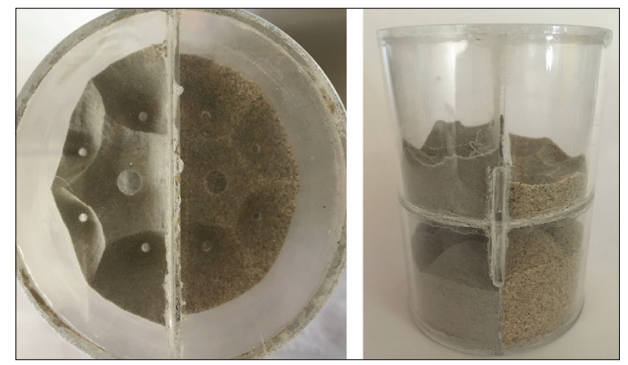

Overall dimensions of the device are the same as before, but a vertical plate divides each of the lower and upper chambers into two equal bins, allowing for separation of the two granular materials. The separating disk between the upper and lower chambers has symmetrical holes on both sides of the vertical divide.

When the cylinder is turned upside down, sand and CMS flow through identical geometries but very differently. The sand flows quickly and uniformly, as shown in the video, ${ }^{2}$ while the CMS, as described previously, flows erratically and exhibits intermittent landslide- and eruption-like flows. The main reason for the difference is cohesion among the CMS grains. The photo on the right shows how more of the sand gets through the holes even though it has larger grain size.

1. S. Shakerin, "Fluid demonstrations II: Bubbles in Mondrian painting, eruption-like flow, rotational instability, and wake vortices," Phys. Teach. 57, 600-603 (Dec. 2019).

2. Readers can view the video at TPT Online, http://dx.doi. org/10.1119/1.5145398, under the Supplemental tab.

Said Shakerin

University of the Pacific sshakerin@pacific.edu

THE PHYSICS TEACHER • VOI. 58, MARCH 2020 